\section{Cahiers de littérature orale}

67-68 | 2010

Quand l'art prend la parole

\title{
Michèle THERRIEN (dir.), Paroles interdites
}

\section{Cécile Leguy}

\section{OpenEdition \\ Journals}

\section{Édition électronique}

URL : https://journals.openedition.org/clo/855

DOI : $10.4000 /$ clo.855

ISSN : 2266-1816

Éditeur

INALCO

\section{Édition imprimée}

Date de publication : 15 janvier 2010

Pagination : 273-276

ISBN : 978-2-85831-196-5

ISSN : 0396-891X

\section{Référence électronique}

Cécile Leguy, « Michèle Therrien (dir.), Paroles interdites », Cahiers de littérature orale [En ligne], 67-68| 2010, mis en ligne le 01 mars 2013, consulté le 30 juin 2021. URL : http://journals.openedition.org/ clo/855 ; DOl : https://doi.org/10.4000/clo.855

Ce document a été généré automatiquement le 30 juin 2021.

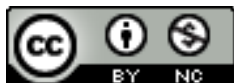

Cahiers de littérature orale est mis à disposition selon les termes de la Licence Creative Commons Attribution - Pas d'Utilisation Commerciale 4.0 International. 


\title{
Michèle THERRIEN (dir.), Paroles interdites
}

\author{
Cécile Leguy
}

\section{RÉFÉRENCE}

Michèle THERRIEN (dir.), Paroles interdites, Paris, Karthala, 2008, 288 p. ISBN :

978-2-8111-0031-5.

1 Rassemblant en onze chapitres une réflexion sur l'interdit langagier, l'ouvrage dirigé par Michèle Therrien nous invite à évaluer, à partir de contextes culturels divers, les comportements verbaux en pratique et les moyens offerts par les détours du langage afin de se faire entendre tout en préservant la paix sociale. « Trop parler peut s'avérer nuisible», nous précise Michel Aufray au sujet des sociétés océaniennes. Cette remarque pourrait bien s'avérer valoir en tout lieu, en tout temps... C'est du moins ce qu'on peut retenir de cette exploration qui nous mène des sociétés du Pacifique aux Inuit, en passant par la Thaïlande, l'Inde, les Philippines, l'Afrique et l'Europe.

2 Les paroles interdites, ce sont d'abord tous ces mots qu'il ne sied pas de prononcer, parce qu'ils relèvent de domaines particuliers, avec lesquels le langage entretient des rapports délicats, comme celui de la sexualité ou bien des excrétions corporelles, ou encore les domaines des émotions, de la douleur, ceux de la chasse, de la guerre et de la mort, celui des symboles politiques ou religieux et même de la météo (chez les Inuit, voir la contribution de Michèle Therrien). Les interdits touchant à ces divers domaines semblent traverser les différentes sociétés, avec des accents plus ou moins forts selon les cultures. Les auteurs présentent les recours langagiers qui permettent de contourner, et même de transgresser parfois, les paroles interdites. Le recours à l'antiphrase par exemple, dont usent les Inuit quand ils disent «quel temps magnifique!" pour ne pas se plaindre du blizzard, est une manière d'exprimer son dépit de ne pouvoir sortir sans prendre de risque. La plaisanterie, qu'il est possible de partager avec certaines personnes, de manière plus ou moins codifiée selon les sociétés, 
permet d'exprimer face à certains les paroles osées qu'on retient face aux autres. Les relations à plaisanterie entretenues entre certains individus bien précis, qu'on se situe en Océanie (Aufray), en Afrique (Calame-Griaule) ou en Alaska (Therrien), jouent bien souvent le rôle d'exutoire, dans des conditions où l'efficacité des "mauvaises paroles » est contrée par la situation particulière qui lie les interlocuteurs. Le déguisement lexical, tel qu'on le trouve dans nos anciennes formules blasphématoires en français par exemple (p. 35), est également un moyen détourné de dire sans dire, de ne pas « vraiment » prononcer les paroles interdites et, ainsi, d'en éviter les dangers.

3 Injures et insultes sont à l'inverse une manière de recourir à l'énonciation de paroles interdites, dans un cadre bien précis, même si c'est parfois par le biais d'euphémismes. Dans certains cas, comme on peut le voir dans les contributions de Michel Aufray et de Philippe Benoît, le discours peut alors être très cru, très direct, et les "paroles interdites » émises de manière éhontée sont là pour provoquer l'interlocuteur, lui faire violence. Mais le plus souvent, la formule est d'autant plus percutante qu'elle est évasive et même poétique, comme le montre bien l'exemple inuit où l'on recourt plutôt à la litote pour exprimer son mécontentement. Comme le disait bien Évelyne Larguèche : «L'injure est dans la relation que crée son énonciation et non dans l'énoncé auquel elle fait croire $»^{1}$ et il est souvent plus efficace de laisser entendre les paroles interdites plutôt que de s'abaisser à les énoncer. Car l'injure n'est pas seulement une parole visant à exprimer son mécontentement, elle est une formule qui peut agir sur la personne à qui elle s'adresse, à l'instar de ces enfants blâmés dans la tradition slave orientale qui, selon les circonstances de l'énonciation de la mauvaise parole, peuvent effectivement être " pris par le diable » et voir leur vie gâchée (voir la contribution de Galina Kabakova). Ainsi Michel Aufray précise-t-il que la plus grande injure en contexte océanien serait de prétendre avoir oublié le nom de quelqu'un, ce qui correspondrait à nier son existence.

4 Les noms des personnes ou des entités religieuses sont effectivement bien souvent l'objet d'interdits, ce qu'on retrouve dans différentes contributions où sont mentionnées diverses stratégies permettant de désigner ou de s'adresser à quelqu'un sans prononcer son nom. Par exemple, l'usage très répandu de la teknonymie, qui offre notamment aux femmes la possibilité de désigner leur mari dès qu'elles ont enfanté, en évitant la honte d'avoir à prononcer son nom propre ou encore de contrer les moqueries quand on feint de ne pas la comprendre, parce qu'elle parle de lui de manière trop évasive (voir la contribution de Geneviève Calame-Griaule). Les noms de personnes font couramment l'objet d'interdits, en adresse comme en référence, en fonction des relations sociales qui lient les personnes, notamment de cadets à aînés par exemple, ou encore entre alliés. Des stratégies peuvent alors permettre de détourner ces interdictions, comme la dation réciproque d'un surnom dyadique (laläw) chez les Palawan des Philippines, choisi à l'occasion d'un événement comique vécu en partage. Le surnom réciproque est alors substitué en référence et en adresse au nom comme au terme de parenté, ce qui peut permettre d'échapper à l'interdiction d'énonciation de l'un comme de l'autre (voir la contribution de Nicole Revel). Si le poète se masque derrière un nom de plume, comme c'est le cas en poésie classique ourdou (ghazal) où il reçoit celui-ci de son maître (Pakistan et nord de l'Inde, voir la contribution d'Alain Désoulières), il peut également refuser de dévoiler son identité d'auteur quand il se sent en situation délicate en ne récitant pas le poème en entier, le dernier vers contenant justement le nom de plume par lequel il est connu. 
L'expression littéraire est souvent un moyen détourné d'enfreindre les interdits. C'est le cas de la poésie chantée, telle qu'elle apparaît au cœur du cinéma indien présenté par Alain Désoulières par exemple, ou encore dans les chants d'amour alternés entre homme(s) et femme(s) recueillis auprès des Palawan des montagnes philippines par Nicole Revel, qui expriment avec finesse et retenue les paroles imprononçables de la passion amoureuse. Poésie et musique offrent la possibilité de faire entendre des paroles qui ne sauraient se dire autrement, comme ces chants inuit à forte connotation sexuelle (iviutiit) énoncés lors des rassemblements festifs, dans un contexte où pudeur et retenue sont de mise, surtout en présence des enfants.

6 Quelques contributions ouvrent des pistes de réflexion sur l'interdiction de parole et ses rapports avec la réalité. Que signifie en effet un interdit reposant sur l'énonciation de mots, si ce n'est la valeur pragmatique accordée à celle-ci ? C'est ce que montrent bien les contes de la tradition orale européenne présentant des cas de "paroles interdites » analysés par Nicole Belmont. Si refuser la communication langagière, c'est aussi refuser comme le disait Lévi-Strauss - convoqué ici par Belmont - d'autres types de communication, ce refus oblige à s'interroger sur les relations entre les mots et les choses, entre ce qui est partagé par la parole et ce qui est autrement vécu dans les relations sociales, amoureuses ou conflictuelles et ne saurait être partagé n'importe comment, ni avec n'importe qui. De même, Geneviève Calame-Griaule ne se contente pas de lister les euphémismes tels qu'ils sont utilisés dans la parole quotidienne en pays dogon, mais cherche à appréhender la conception du phénomène langagier qu'ils soustendent. "Comment le fait de remplacer un nom par un autre atténue-t-il sa portée ?", s'interroge-t-elle (p. 158). Ne s'agit-il pas ici, comme on peut le faire dans d'autres contextes, de cacher tout en montrant, de mieux montrer en cachant, d'accéder au sens par le biais efficace du détour ${ }^{2}$ ? Si les paroles interdites trouvent malgré tout des chemins détournés pour être entendues, n'est-ce pas, ainsi que le conçoivent les Inuit, en raison de l'aspect néfaste de ces paroles quand elles demeurent dans la pensée, mettant en danger la santé même de celui qui les retient?

7 Le pouvoir de la parole est plus manifeste encore quand elle touche aux désirs interdits: jalousie, envie de meurtre ou de vengeance, comme c'est le cas dans le contexte de la parole magique et même de la sorcellerie, où l'enquêteur lui-même est confronté à la rétention de parole. Comme le montre bien Micheline Lebarbier en contexte roumain, les «paroles interdites » dans ce domaine ne sont pas seulement les paroles néfastes de l'attaque ou de l'envoûtement, mais aussi les paroles inverses du désenvoûtement qu'il est délicat d'avouer connaître, dans la mesure où cela reviendrait à admettre sa maîtrise du rituel inverse. Dans le cadre de ces pratiques langagières éminemment performatives, le savoir est d'autant plus efficace qu'il a été acquis en cachette et non par une transmission directe, les "paroles interdites» étant plus puissantes encore quand elles sont dérobées à l'insu de celui qui les prononce.

8 Ces "paroles interdites", consignées ici dans un ouvrage collectif où elles sont appelées à dévoiler une part de leur mystère, nous invitent ainsi, au-delà des données ethnographiques et littéraires convoquées dans les différentes contributions, à une réflexion plus générale sur les pouvoirs et les dangers des mots, ainsi que sur les manières d'en user ou de s'en prémunir, selon les contextes. 


\section{NOTES}

1. Évelyne Larguèche, L'injure comme objet anthropologique, Revue des mondes musulmans et de la Méditerranée, $\mathrm{n}^{\circ}$ 103/104, 2004, p. 36.

2. Cf. François Jullien, Le détour et l'accès. Stratégies du sens en Chine, en Grèce, Paris, Grasset, 1995. 